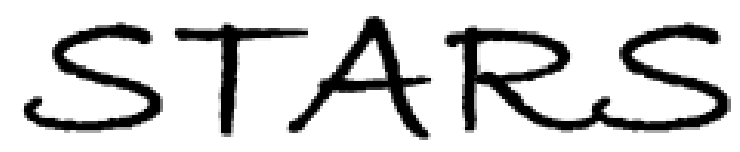

University of Central Florida

STARS

$1-1-2008$

\title{
Propagation of Local Disturbances in Reaction Diffusion Systems Modeling Quadratic Autocatalysis
}

Xinfu Chen

Yuanwei Qi

University of Central Florida

Find similar works at: https://stars.library.ucf.edu/facultybib2000 University of Central Florida Libraries http://library.ucf.edu

This Article is brought to you for free and open access by the Faculty Bibliography at STARS. It has been accepted for inclusion in Faculty Bibliography 2000s by an authorized administrator of STARS. For more information, please contactSTARS@ucf.edu.

\section{Recommended Citation}

Chen, Xinfu and Qi, Yuanwei, "Propagation of Local Disturbances in Reaction Diffusion Systems Modeling Quadratic Autocatalysis" (2008). Faculty Bibliography 2000s. 213.

https://stars.library.ucf.edu/facultybib2000/213

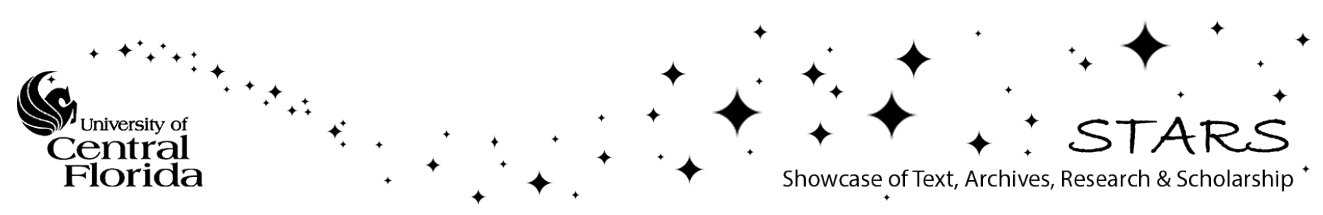




\title{
PROPAGATION OF LOCAL DISTURBANCES IN REACTION DIFFUSION SYSTEMS MODELING QUADRATIC AUTOCATALYSIS*
}

\author{
$\mathrm{XINFU} \mathrm{CHEN}^{\dagger}$ AND YUANWEI $\mathrm{QI}^{\ddagger}$
}

\begin{abstract}
This article studies the propagation of initial disturbance in a quadratic autocatalytic chemical reaction in one-dimensional slab geometry, where two chemical species $A$, called the reactant, and $B$, called the autocatalyst, are involved in the simple scheme $A+B \rightarrow 2 B$. Experiments demonstrate that chemical systems for which quadratic or cubic catalysis forms a key step can support propagating chemical wavefronts. When the autocatalyst is introduced locally into an expanse of the reactant, which is initially at uniform concentration, the developing reaction is often observed to generate two wavefronts, which propagate outward from the initial reaction zone. We show rigorously that with such an initial setting the spatial region is divided into three regions by the two wavefronts. In the middle expanding region, the reactant is almost consumed so that $A \approx 0$, whereas in the other two regions there is basically no reaction so that $B \approx 0$. Most of the chemical reaction takes place near the wavefronts. The detailed characterization of the concentrations is given for each of the three zones.
\end{abstract}

Key words. quadratic autocatalysis, traveling wave, propagation of local disturbance, reactiondiffusion

AMS subject classifications. $34 \mathrm{C} 20,34 \mathrm{C} 25,92 \mathrm{E} 20$

DOI. $10.1137 / 07070276 \mathrm{X}$

1. Introduction. In this paper we consider an isothermal autocatalytic chemical reaction step governed by the quadratic reaction relation

$$
A+B \rightarrow 2 B \text { with rate } k a b .
$$

Here, $k>0$ is the reaction rate, and $a$ and $b$ are the concentrations of reactant $A$ and autocatalyst $B$, respectively.

Well documented in the literature, the quadratic reaction relation has appeared in several important models of real chemical reactions, e.g., the Belousor-Zhabotinskii reaction and also gas-phase radical chain branching, oxidation reactions, such as the carbon-monoxide-oxygen reaction, and hydrogen-oxygen systems [13].

Experimental observations demonstrate the existence of propagating chemical wave fronts in unstirred chemical systems for which quadratic or cubic catalysis forms a key step [15], [25]. These wavefronts, or travelling waves, arise due to the interaction of reaction and diffusion. Quite often when a quantity of autocatalyst is added locally into an expanse of reactant, which is initially at uniform concentration, the ensuing reaction is observed to generate wavefronts which propagate outward from the initial reaction zone, consuming fresh reactant ahead of the wavefront as it propagates. This is the phenomenon to be addressed in this paper.

* Received by the editors September 13, 2007; accepted for publication (in revised form) March 31, 2008; published electronically October 13, 2008.

http://www.siam.org/journals/siap/69-1/70276.html

${ }^{\dagger}$ Department of Mathematics, University of Pittsburgh, Pittsburgh, PA 15260 (xinfu@pitt.edu). The work of this author was supported by National Science Foundation grant DMS-0504691.

${ }^{\ddagger}$ Department of Mathematics, University of Central Florida, Orlando, FL 32816 (yqi@pegasus.cc. ucf.edu). The work of this author was supported by National Science Foundation grant DMS-0504691 during his visit to the University of Pittsburgh. 
We study the following system for $u=u(x, t), v=v(x, t)$ :

$$
\begin{cases}u_{t}-D u_{x x}=-u v & \text { in } \mathbb{R} \times(0, \infty), \\ v_{t}-v_{x x}=u v & \text { in } \mathbb{R} \times(0, \infty), \\ u(\cdot, 0)=u_{0}(\cdot), \quad v(\cdot, 0)=v_{0}(\cdot) & \text { on } \mathbb{R} \times\{0\}\end{cases}
$$

It is the result of simple scaling of the standard system

$$
a_{t}=D_{A} a_{x x}-k a b, \quad b_{t}=D_{B} b_{x x}+k a b,
$$

with $D=D_{A} / D_{B}$.

Our basic assumptions are the following:

(A1) $D \in(0,1]$;

(A2) $u_{0}(x)=1$ for all $x \in \mathbb{R}$; and

(A3) $v_{0}$ is a continuous nonnegative function having compact support, $v_{0}(0)>0$.

Our main result is the following.

TheOrem 1.1. Assume (A1)-(A3) and let $(u, v)$ be the solution of (1.1). Set

$$
m(t)=2 t-3(\log [3+t]-\log 3) .
$$

Then for each $t>0$ and $x \in[-m(t), m(t)]$, we have $(u, v) \approx(0,1)$ in the following sense:

$$
u(x, t) \leq e^{-\mu[m(t)-|x|]}, \quad|1-v(x, t)| \leq \frac{C}{\sqrt{1+m(t)-|x|}} .
$$

On the other hand, when $x \in(-\infty,-m(t)] \cup[m(t), \infty)$, we have $(u, v) \approx(1,0)$ in the sense that

$$
|1-u(x, t)|+v(x, t) \leq C\{1+|x|-m(t)\} e^{m(t)-|x|} .
$$

A result somewhat similar to ours is obtained by Billingham and Needham [7] using formal asymptotic and numerical computation. There, instead of a Cauchy initial problem, an initial-boundary value problem on $(0, \infty)$ is considered, with a homogenous Newmann condition at $x=0$. The proof we give here is rigorous.

It will be interesting to see how to generalize our result to the cubic autocatalysis reaction with nonlinear reaction term $u v^{2}$. But a number of technical difficulties need to be overcome, not least of which is a result similar to that of Bramson on the traveling speed of a scalar equation with nonlinearity $u(1-u)^{2}$.

The organization of this paper is as follows. Section 2 contains the analysis of $u$ behind the reaction front. In section 3 the estimate of the front location is provided. The behavior of $(u, v)$ after the reaction has taken place is shown in section 4 .

We note in passing that unlike the single equation case, of which many excellent results have been proved in the last 30 years as exemplified by the works of Aronson and Weinberger [2], Fife and McLeod [10], Sattinger [21], and Chen and Guo [8] (the survey paper of Xin [24] provides a more detailed account on recent progress), there are very limited results on the study of traveling waves and their effect on global dynamics for parabolic systems. With the recent progress of proving the existence of traveling waves in [9] and [20], we hope to spur interest in such problems since many mathematical models in biology, most of which are reaction-diffusion systems, are deeply linked to traveling wave phenomena. We also note that systems similar to ours appear in the study of thermal-diffusive flows with advection; see [4], [16], [17], [18], [19], and [23]. 
2. Exponential decay of a reactant behind a reaction front. Whenever an autocatalyst presents, the chemical reaction takes place very fast; as a result, the reactant is consumed quickly and therefore experiences an exponential decay (in time). The central issue here is to find the spreading speed of the autocatalyst. Mathematically, by assuming $D \in(0,1]$ (i.e., the reactant diffuses no faster than the autocatalyst does), we are able to find a good comparison to pin down the autocatalyst's spreading speed.

\subsection{A comparison.}

Lemma 2.1. Assume that $D \in(0,1]$ and $u_{0}(x) \geq 0, v_{0}(x) \geq 0, u_{0}(x)+v_{0}(x) \geq 1$ for every $x \in \mathbb{R}$. Then the solution of (1.1) satisfies

$$
v(x, t) \geq \sqrt{D} \Phi(x, t) \quad \forall(x, t) \in \mathbb{R} \times(0, \infty),
$$

where $\Phi$ is the solution of the initial value problem of the Fisher KPP (KolmogorovPetrovskii-Piskuno) equation

$$
\Phi_{t}-\Phi_{x x}=\Phi-\Phi^{2} \text { in } \mathbb{R} \times(0, \infty), \quad \Phi(\cdot, 0)=v_{0}(\cdot) \quad \text { on } \mathbb{R} \times\{0\} .
$$

Proof. Denote by $K(x, t)$ the fundamental solution to the heat operator,

$$
K(x, t):=(4 \pi t)^{-1 / 2} e^{-x^{2} /(4 t)} .
$$

Then the solution of (1.1) can be decomposed as

$$
u=u^{0}-u^{1}, \quad v=v^{0}+v^{1}
$$

where

$$
\begin{aligned}
u^{0}(x, t) & =\int_{\mathbb{R}} K(x-y, D t) u_{0}(y) d y, \\
v^{0}(x, t) & =\int_{\mathbb{R}} K(x-y, t) v_{0}(y) d y, \\
u^{1}(x, t) & =\int_{0}^{t} \int_{\mathbb{R}} K(x, y, D(t-s)) f(y, s) d y d s, \\
v^{1}(x, t) & =\int_{0}^{t} \int_{\mathbb{R}} K(x-y, t-s) f(y, s) d y d s, \\
f(x, t) & =u(x, t) v(x, t) .
\end{aligned}
$$

Here $u^{0}$ and $v^{0}$ are the concentrations of the reactant and the autocatalyst, respectively, before chemical reaction is initiated. The quantity $u^{1}$ is the amount of reactant consumed and $v^{1}$ is the amount of autocatalyst produced in the reaction.

By the maximum principle, we know that $u \geq 0$ and $v \geq 0$, and so $f:=u v \geq 0$. Upon noticing that

$$
K(x, D t):=(4 \pi D t)^{-1 / 2} e^{-x^{2} /(4 D t)} \leq(4 \pi D t)^{-1 / 2} e^{-x^{2} /(4 t)}=D^{-1 / 2} K(x, t),
$$

we see that

$$
u^{1}(x, t) \leq D^{-1 / 2} v^{1}(x, t) \quad \forall(x, t) \in \mathbb{R} \times[0, \infty) .
$$

This implies that

$$
u=u^{0}-u^{1} \geq u^{0}-\frac{v^{1}}{\sqrt{D}}=u^{0}-\frac{v-v^{0}}{\sqrt{D}}=\left(u^{0}+\frac{v^{0}}{\sqrt{D}}\right)-\frac{v}{\sqrt{D}} .
$$

Copyright (c) by SIAM. Unauthorized reproduction of this article is prohibited. 
Note that

$$
\begin{aligned}
u^{0}(x, t)+\frac{v^{0}(x, t)}{\sqrt{D}} & =\int_{\mathbb{R}} K(y, D t) u_{0}(x-y) d y+\frac{1}{\sqrt{D}} \int_{\mathbb{R}} K(y, t) v_{0}(x-y) d y \\
& =\frac{1}{\sqrt{\pi}} \int_{\mathbb{R}}\left\{e^{-\eta^{2}} u_{0}(x-2 \eta \sqrt{D t})+e^{-D \eta^{2}} v_{0}(x-2 \eta \sqrt{D t})\right\} d \eta \\
& \geq \frac{1}{\sqrt{\pi}} \int_{\mathbb{R}} e^{-\eta^{2}}\left\{u_{0}(x-2 \eta \sqrt{D t})+v_{0}(x-2 \eta \sqrt{D t})\right\} d \eta \\
& \geq \frac{1}{\sqrt{\pi}} \int_{\mathbb{R}} e^{-\eta^{2}} d \eta=1
\end{aligned}
$$

Thus,

$$
\left(\frac{v}{\sqrt{D}}\right)_{t}-\left(\frac{v}{\sqrt{D}}\right)_{x x}=u \frac{v}{\sqrt{D}} \geq\left(1-\frac{v}{\sqrt{D}}\right) \frac{v}{\sqrt{D}}
$$

A simple comparison then gives $\Phi \leq v / \sqrt{D}$.

2.2. Bramson's result. We denote by $W$ the minimum speed traveling wave profile of the Fisher equation

$$
\begin{gathered}
2 W^{\prime}+W^{\prime \prime}+W-W^{2}=0 \quad \text { on } \mathbb{R}, \\
W(-\infty)=1, \quad W(0)=1 / 2, \quad W(\infty)=0 .
\end{gathered}
$$

The following result can be derived from Bramson's work [3].

Lemma 2.2. Assume that $v_{0}$ is a nonnegative continuous function on $\mathbb{R}$ with compact support and $v_{0}(0)>0$. Let $\Phi$ be the solution of (2.1). Then there exist constants $z_{+}$and $z_{-}$such that

$$
\begin{array}{ll}
\lim _{t \rightarrow \infty} \sup _{x>0}\left|\Phi(x, t)-W\left(\left[x-z_{+}-m(t)\right]\right)\right|=0, \\
\lim _{t \rightarrow \infty} \sup _{x<0}\left|\Phi(x, t)-W\left(\left[m(t)+z_{-}-x\right]\right)\right|=0,
\end{array}
$$

where

$$
m(t):=2 t-3[\log (3+t)-\log 3] \quad \forall t>0 .
$$

\subsection{The exponential decay of $\boldsymbol{u}$ in the reaction zone.}

Theorem 2.3. Assume that $D \in(0,1], u_{0} \geq 0, v_{0} \geq 0, u_{0}+v_{0} \geq 1$, and $v_{0}(0)>0$. Let $(u, v)$ be the solution of (1.1). Then there exists a positive constant $k$ such that

$$
v>k \quad \text { in } Q:=\{(x, t)|t>0,| x \mid<m(t)\} .
$$

Consequently, with $\mu=[\sqrt{1+k D}-1] / D$, there holds

$$
u(x, t) \leq \bar{u}(x, t):=e^{\mu[x-m(t)]}+e^{-\mu[m(t)+x]} \quad \forall t \geq 0, x \in \mathbb{R} .
$$

Proof. First, applying the comparison lemma, Lemma 2.1, and Bramson's result, Lemma 2.2, we see that $v>k$ in $Q$.

Copyright $@$ by SIAM. Unauthorized reproduction of this article is prohibited. 
Since $u \leq 1$, we need only consider the function $u$ in the set $Q$. When $(x, t) \in Q$, we use $v \geq k$ to calculate

$$
\begin{aligned}
\bar{u}_{t} & -D \bar{u}_{x x}+v \bar{u} \geq \bar{u}_{t}-D \bar{u}_{x x}+k \bar{u} \\
& =\bar{u}\left\{k-D \mu^{2}-2 \mu+\frac{3 \mu}{3+t}\right\} \geq \bar{u}\left[k-D \mu^{2}-2 \mu\right]=0 .
\end{aligned}
$$

Since $\bar{u}>1 \geq u$ on the parabolic boundary of $Q$, the assertion of the lemma thus follows from the parabolic comparison principle.

3. Location of the reaction front. The comparison of $v$ with the solution of the Fisher equation shows that the reaction front is at least as far as $\pm(2 t-3 \log t)$ from the origin for large $t$. Here we show that the reaction front is located exactly in a vicinity of $\pm(2 t-3 \log t)$.

For this, we denote

$$
\hat{u}(x, t)=\min \left\{1, e^{\mu[x-m(t)]}+e^{-\mu[m(t)+x]}\right\} .
$$

Then $u \leq \hat{u}$. Consequently,

$$
v_{t}-v_{x x}=u v \leq \hat{u} v \quad \text { in } \mathbb{R} \times(0, \infty) .
$$

Hence, by Green's formula,

$$
0 \leq v(x, t) \leq \int_{\mathbb{R}} G(x, t ; y, 0) v_{0}(y) d y,
$$

where for each $(x, t) \in \mathbb{R} \times(0, \infty), G(x, t ; \cdot, \cdot)$ is the fundamental solution of

$$
\begin{aligned}
G_{s}+G_{y y}=\hat{u}(y, s) G(x, t, y, s) & \forall y \in \mathbb{R}, s \in[0, t), \\
G(x, t ; y, t)=\delta(x-y) & \forall y \in \mathbb{R} .
\end{aligned}
$$

Here $\delta$ is the Dirac measure. Using Bramson's technique [3, Chapters 6 and 7], one can derive that

$$
G(x, y, t, 0) \leq \frac{C(\mu) e^{t-|x-y|^{2} /(4 t)}}{\sqrt{4 \pi t}}\left(1-e^{-|y|[|x|-m(t)+1] / t}\right) .
$$

Since $v_{0}$ has compact support, by following calculations illustrated in [3] we obtain the following.

Lemma 3.1. There exists a positive constant $C_{1}$ such that

$$
v( \pm[m(t)+z], t) \leq C_{1}[1+|z|] e^{-z} \quad \forall z \in \mathbb{R}, t>0 .
$$

Note that when $u_{0} \equiv 1$, we have $u^{0} \equiv 1$ so that

$$
|u-1|=u^{1} \leq D^{-1 / 2} v^{1} \leq D^{-1 / 2} v .
$$

The estimate (1.4) thus follows from the above lemma.

4. Autocatalyst generated after reaction. We know that the two reaction fronts are near $m(t)$ and $-m(t)$. In the reaction zone $[-m(t), m(t)]$, the reactant is consumed very quickly. As the autocatalyst is assumed to diffuse no slower than the reactant, it is expected that $v \approx 1$ inside the reaction zone when reaction is completed. This section is devoted to proving this expectation.

Copyright (c) by SIAM. Unauthorized reproduction of this article is prohibited. 


\subsection{An $L^{\infty}$ estimate of $v$.}

Lemma 4.1. There exists a positive constant $C_{2}$ such that

$$
v(x, t) \leq C_{2}, \quad\left|u_{x}\right| \leq C_{2} e^{-\mu|| x|-m(t)|]} \quad \forall x \in \mathbb{R}, t>0 .
$$

Proof. Set

$$
K=\max \left\{\frac{1}{4}, \frac{1}{\left(\mu^{2}+2 \mu\right)}\right\} .
$$

Let $t_{0}$ be the constant such that

$$
K e^{-m\left(t_{0}\right)}=\frac{1}{4}
$$

Consider the function

$$
\bar{v}(x, t)=1-K \bar{u}=1-K e^{\mu[x-m(t)]}-K e^{-\mu[x+m(t)]}
$$

in the set

$$
Q\left(t_{0}\right):=\left\{(x, t)\left|t>t_{0},\right| x \mid<m(t)-m\left(t_{0}\right)\right\} .
$$

Since $u<\bar{u}$ in $Q\left(t_{0}\right)$, we have

$$
\bar{v}_{t}-\bar{v}_{x x}-u \bar{v} \geq \bar{v}_{t}-\bar{v}_{x x}-\bar{u} \bar{v} \geq K \bar{u}^{2}>0 .
$$

Then we have $\bar{v} \geq 1 / 2$ on the parabolic boundary of $Q\left(t_{0}\right)$. Hence, by comparison,

$$
v \leq M \bar{v} \text { in } Q\left(t_{0}\right), \quad M:=\sup _{\partial Q\left(t_{0}\right)} v \leq C_{1}\left[1+m\left(t_{0}\right)\right] e^{m\left(t_{0}\right)} .
$$

This estimate, together with Lemma 3.1, implies that $v$ is uniformly bounded.

Once we know the boundedness of $v$, we can obtain the estimate for $u_{x}$ by applying the local parabolic estimate. For each $x \in \mathbb{R}$ and $t \geq 2$,

$$
\left\|u_{x}\right\|_{L^{\infty}\left(Q_{1}\right)} \leq C(D)\left\{\|f\|_{L^{\infty}\left(Q_{2}\right)}+\min \left\{\|u\|_{L^{\infty}\left(Q_{2}\right)},\|u-1\|_{L^{\infty}\left(Q_{2}\right)}\right\}\right\},
$$

where

$Q_{1}=(x-1, x+1) \times(\max \{t-1,0\}, t], \quad Q_{2}:=(x-2, x+2) \times(\max \{t-2,0\}, t]$.

Here we used, for simplicity, the assumption that $u_{0} \equiv 1$ is a smooth function.

4.2. The equilibrium state after reaction. Now we show that $v \approx 1$ in $(-m(t), m(t))$ for large $t$. For this purpose, we consider the function

$$
w=u+v-u^{0}-v^{0} .
$$

Note that $u^{0} \equiv 1$; then

$$
\begin{aligned}
&\left\|v^{0}(\cdot, t)\right\|_{L^{\infty}(\mathbb{R})}=\left\|\int_{\mathbb{R}} K(\cdot-y, t) v_{0}(y) d y\right\|_{L^{\infty}(\mathbb{R})}=O\left(\frac{1}{\sqrt{t}}\right), \\
&|u(x, t)| \leq e^{-\mu|m(t)-x|}+e^{-\mu[x+m(t)]} .
\end{aligned}
$$

Copyright $@$ by SIAM. Unauthorized reproduction of this article is prohibited. 
We see that

$$
|v-1| \leq|w|+|u|+\left|v^{0}\right| .
$$

The assertion (1.3) thus follows from the following.

Lemma 4.2. There exists a constant $C_{2}>0$ such that

$$
|w(x, t)| \leq \frac{C_{2}}{\sqrt{m(t)-|x|}} \quad \forall x \in(-m(t), m(t)), t>0 .
$$

Proof. Note that $w$ satisfies

$$
w_{t}-w_{x x}=(D-1) u_{x x} \quad \text { in } \mathbb{R} \times(0, \infty), \quad w(\cdot, 0)=0 .
$$

Hence,

$$
\begin{aligned}
w(x, t) & =(D-1) \int_{0}^{t} \int_{\mathbb{R}} K(x-y, t-s) u_{y y}(y, s) d y d s \\
& =(D-1) \int_{0}^{t} \int_{\mathbb{R}} K_{x}(x-y, t-s) u_{y}(y, s) d y d s .
\end{aligned}
$$

It then follows that

$$
|w(x, t)| \leq C(1-D)\{J(x, t)+J(-x, t)\}
$$

where

$$
\begin{aligned}
J(x, t) & =\int_{0}^{t} \int_{\mathbb{R}}\left|K_{x}(x-y, t-s)\right| e^{-\mu|y-m(s)|} d y d s \\
& =\int_{0}^{t} \int_{\mathbb{R}}\left|K_{x}(x-y-m(t-s), s)\right| e^{-\mu|y|} d y d s .
\end{aligned}
$$

To complete the proof, it suffices to show the following:

$$
J(m(t)-z, t) \leq \frac{C}{\sqrt{z}} \quad \forall z>0 .
$$

Let $z>0$ and $t>0$ be arbitrary. Note that

$$
\begin{aligned}
J(m(t)-z, t) & =\int_{0}^{t} \int_{\mathbb{R}}\left|K_{x}(m(t)-m(t-s)-z-y, s)\right| e^{-\mu|y|} d y d s \\
K_{x}(x, s) & =-\frac{x e^{-x^{2} /(4 s)}}{4 \sqrt{\pi} s^{3 / 2}} .
\end{aligned}
$$

We divide the integral in $s$ into the following three intervals.

(i) $s \in[z / 4,2 z]$. For each fixed $y \in \mathbb{R}$, we have

$$
\begin{aligned}
& \int_{z / 4}^{2 z}\left|K_{x}(m(t)-m(s)-z-y, s)\right| d s \\
\leq & \int_{z / 4}^{2 z} \frac{|m(t)-m(t-s)-y-z|}{4 \sqrt{\pi}[z / 4]^{3 / 2}} e^{-|m(t)-m(t-s)-y-z|^{2} /(4 z)} d s .
\end{aligned}
$$

Copyright (c) by SIAM. Unauthorized reproduction of this article is prohibited. 
We use the change of variable from $s$ to $\eta$ defined by

$$
\eta=\frac{m(t)-m(t-s)-z-y}{z}, \quad d \eta=\frac{m^{\prime}(t-s)}{z} d s=\frac{2-\frac{3}{3+(t-s)}}{z} d s \geq \frac{d s}{z} .
$$

We find that

$$
\int_{z / 4}^{\min \{2 z, t\}}\left|K_{x}(m(t)-m(t-s)-z-y, s)\right| d s \leq \frac{2}{\sqrt{\pi z}} \int_{\mathbb{R}} \eta e^{-\eta^{2}} d \eta=\frac{2}{\sqrt{\pi z}} .
$$

It then follows that

$$
\int_{z / 4}^{\min \{2 z, t\}} \int_{\mathbb{R}}\left|K_{x}(m(t)-m(t-s)-z-y, s)\right| e^{-\mu|y|} d s d y \leq \frac{2}{\sqrt{\pi z}} \int_{\mathbb{R}} e^{-\mu|y|} d y \leq \frac{4}{\mu \sqrt{\pi z}}
$$

(ii) $s>2 z$. We write

$$
\int_{\mathbb{R}}\left|K_{x}(m(t)-m(t-s)-z-y, s)\right| e^{-\mu|y|} d y=\int_{|y|>s / 6}+\int_{|y|<s / 6} .
$$

For the first integral,

$$
\begin{aligned}
\int_{|y|>s / 6} & \leq e^{-\mu s / 6} \int_{\mathbb{R}} \mid K_{x}(m(t)-m(t-s)-z-y, s \mid d y \\
& =2 e^{-\mu s / 6} K(0, s)=\frac{e^{-\mu s / 6}}{\sqrt{\pi s}} .
\end{aligned}
$$

For the second integral, we first notice that $|m(t)-m(t-s)| \geq s$ (since $1 \leq m^{\prime}<2$ on $[0, \infty))$. Hence, when $|y|<s / 6$,

$$
|m(t)-m(t-s)-z-y| \geq|m(t)-m(t-s)|-z-y \geq s-\frac{s}{2}-\frac{s}{6}=\frac{s}{3} .
$$

Consequently,

$$
\int_{|y|<s / 6}\left|K_{x}\right| e^{-\mu|y|} d y \leq \int_{|x|>s / 3}\left|K_{x}(x, s)\right| d x=2 K\left(\frac{s}{3}, s\right)=\frac{\sqrt{3} e^{-s / 36}}{\sqrt{\pi s}} .
$$

Thus,

$$
\begin{aligned}
\int_{z}^{t} \int_{\mathbb{R}} & \left|K_{x}(m(t)-m(t-s)-z-y, s)\right| e^{-\mu|y|} d y d s \\
& \leq \int_{z}^{\infty}\left(\frac{e^{-\mu s / 6}}{\sqrt{\pi s}}+\frac{\sqrt{3} e^{-s / 36}}{\sqrt{\pi s}}\right) d s=O\left(e^{-z / 36}\right)+O\left(e^{-\mu z / 6}\right) .
\end{aligned}
$$

(iii) $0<s<z / 4$. We write

$$
\int_{\mathbb{R}}\left|K_{x}(m(t)-m(t-s)-z-y, s)\right| e^{-\mu|y|} d y=\int_{|y|>z / 4}+\int_{|y|<z / 4} .
$$

The first integral is easy to estimate:

$$
\int_{|y|>z / 4} \leq e^{-\mu z / 4} \int_{\mathbb{R}}\left|K_{x}\right| d y=\frac{e^{-\mu z / 4}}{\sqrt{\pi s}} .
$$

Copyright (c) by SIAM. Unauthorized reproduction of this article is prohibited. 
For the second integral, we notice that $|m(t)-m(t-s)| \leq 2 s \leq z / 2$, so that when $|y| \leq z / 4$, we have

$$
|m(t)-m(t-s)-z-y| \geq z-|m(t)-m(t-s)|-|y| \geq z-z / 2-z / 4=z / 4 .
$$

Also, $|m(t)-m(t-s)-z-y|<2 z$. Hence,

$$
\left|K_{x}(m(t)-m(t-s)-z-y, s)\right| \leq \frac{z e^{-z^{2} /(64 s)}}{2 \sqrt{\pi} s^{3 / 2}} .
$$

It follows that

$$
\int_{|y|<z / 4}\left|K_{x}\right| e^{-\mu|y|} d y \leq \frac{z e^{-z^{2} /(64 s)}}{2 \sqrt{\pi} s^{3 / 2}} \int_{\mathbb{R}} e^{-\mu|y|}=\frac{z e^{-z^{2} /(64 s)}}{\mu \sqrt{\pi} s^{3 / 2}} .
$$

Thus,

$$
\begin{aligned}
\int_{0}^{\min \{z / 4, t\}} \int_{\mathbb{R}}\left|K_{x}\right| e^{-\mu|y|} d y & \leq \int_{0}^{z / 4} \frac{z e^{-z^{2} /(64 s)} d s}{\mu \sqrt{\pi} s^{3 / 2}}+\int_{0}^{z} \frac{e^{-\mu z / 4} d s}{\sqrt{\pi s}} \\
& =\int_{\sqrt{z / 8}}^{\infty} \frac{4}{\mu \sqrt{\pi}} e^{-\eta^{2}} d \eta+\frac{\sqrt{z} e^{-\mu z / 4}}{\sqrt{\pi}}=O\left(e^{-\mu z / 8}\right) .
\end{aligned}
$$

Combining all these estimate, we then obtain the assertion of the lemma.

Proof of Theorem 1.1. The theorem follows directly from the results of Theorem 2.3 and Lemmas 4.1 and 4.2 .

\section{REFERENCES}

[1] R. Aris, P. Gray, AND S. K. SCOTt, Modelling of cubic autocatalysis by successive biomolecular steps, Chem. Eng. Sci., 43 (1988), pp. 207-211.

[2] D. G. Aronson And H. F. Weinberger, Multidimensional diffusion arising in population genetics, Adv. Math., 30 (1978), pp. 33-76.

[3] M. Bramson, Convergence of solutions of the Kolmogorov equation to travelling waves, Mem. Amer. Math. Soc., 44 (1983), no. 285.

[4] H. Berestycki, F. Hamel, A. Kiselev, and L. RyzhiK, Quenching and propagation in KPP reaction-diffusion equations with a heat loss, Arch. Ration. Mech. Anal., 178 (2005), pp. $57-80$.

[5] J. Billingham and D. J. Needham, The development of travelling wave in quadratic and cubic autocatalysis with unequal diffusion rates. I. Permanent from travelling waves, Philos. Trans. R. Soc. London Ser. A, 334 (1991), pp. 1-24.

[6] J. Billingham and D. J. Needham, The development of travelling wave in quadratic and cubic autocatalysis with unequal diffusion rates. II. An initial value problem with an immobilized or nearly immobilized autocatalyst, Philos. Trans. R. Soc. London Ser. A, 336 (1991), pp. 497-539.

[7] J. Billingham and D. J. Needham, The development of travelling waves in quadratic and cubic autocatalysis with unequal diffusion rates. III. Large time development in quadratic autocatalysis, Quart. Appl. Math., 50 (1992), pp. 343-372.

[8] X. Chen And J.-S. Guo, Existence and asymptotic stability of traveling waves of discrete quasilinear monostable equations, J. Differential Equations, 184 (2002), pp. 549-569.

[9] X. Chen AND Y. QI, Sharp estimates on minimum travelling wave speed of reaction diffusion systems modelling autocatalysis, SIAM J. Math. Anal., 39 (2007), pp. 437-448.

[10] P. C. Fife AND J. B. MCLEOD, The approach of solutions of nonlinear diffusion equations to travelling wave front solutions, Arch. Ration. Mech. Anal., 65 (1977), pp. 335-361.

[11] S. Focant and Th. Gallay, Existence and stability of propagating fronts for an autocatalytic reaction-diffusion system, Phys. D, 120 (1998), pp. 346-368.

[12] R. J. Gowland and G. Stedman, A novel moving boundary reaction involving hydroxylamine and nitric acid, J. Chem. Soc. Chem. Comm., 10 (1983), pp. 1038-1039.

Copyright $@$ by SIAM. Unauthorized reproduction of this article is prohibited. 
[13] P. Gray, J. F. Griffiths, And S. K. Scott, Experiemental studies of the ignition diagram and the effect of added hydrogen, Proc. Roy. Soc. London Ser. A, 397 (1984), pp. 21-44.

[14] P. Gray and S. K. Scott, Chemical Oscillations and Instabilties, Clarendon, Oxford, 1990.

[15] A. Hanna, A. Saul, And K. Showalter, Detailed studies of propagating fronts in the iodate oxidation of arsenous acid, J. Amer. Chem. Soc., 104 (1982), pp. 3838-3844.

[16] S. Heinze, G. Papanicolaou, and A. Stevens, Variational principles for propagation speeds in inhomogeneous media, SIAM J. Appl. Math., 62 (2001), pp. 129-148.

[17] A. Kiselev AND L. RYzhik, Enhancement of the traveling front speeds in reaction-diffusion equations with advection, Ann. Inst. H. Poincaré Anal. Non Linéaire, 18 (2001), pp. 309-358.

[18] B. Khouider, A. Bourlioux, And A. Majda, Parametrizing the burning speed enhancement by small-scale period flows. I. Unsteady shears, flame residence time and bending, Combust. Theory Model., 5 (2001), pp. 295-318.

[19] J. Nolen AND J. XIN, A variational principle based study of KPP minimal front speeds in random shears, Nonlinearity, 18 (2005), pp. 1655-1675.

[20] Y. W. QI, The development of travelling waves in cubic auto-catalysis with different rates of diffusion, Phys. D, 226 (2007), pp. 129-135.

[21] D. Sattinger, On the stability of waves of nonlinear parabolic systems, Adv. Math., 22 (1976), pp. $312-355$.

[22] A. Saul ANd K. Showalter, Propagating reaction-diffusion fronts, in Oscillations and Traveling Waves in Chemical Systems, R. J. Field and M. Burger, eds., Wiley, New York, 1984, pp. 419-439.

[23] J. Shi AND X. WANG, Hair-triggered instability of radial steady states, spread and extinction in semilinear heat equations, J. Differential Equations, 231 (2006), pp. 235-251.

[24] J. XIN, Front propagation in heterogeneous media, SIAM Rev., 42 (2000), pp. 161-230.

[25] A. N. Zaikin And A. M. Zhabotinskil, Concentration wave propagation in two-dimensional liquid-phase self-organising systems, Nature, 225 (1970), pp. 535-537.

Copyright (c) by SIAM. Unauthorized reproduction of this article is prohibited. 